\title{
With a subarachnoid haemorrhage, the outcome is never enough
}

\author{
Wojciech Ambrosius \\ Department of Neurology, Poznan University of Medical Sciences, Poznan, Poland
}

\begin{abstract}
Introduction. In the current edition, Nastasovic et al. present the results of a prospective study on patients with aneurysm subarachnoid haemorrhage $(\mathrm{SAH})$ regarding the association of selected variables and outcomes three months after the incident.

Clinical reflections. The independent predicting factors of an unfavourable aneurysm SAH outcome are aneurysm re-rupture, high systolic blood pressure (SBP), and increased heart rate.

Clinical implications. The article findings confirm easily monitored parameters that could be potentially useful in clinical approaches to this critical illness.
\end{abstract}

(Neurol Neurochir Pol 2019; 53 (6): 398-399)

The increasingly common treatment of ruptured aneurysms with early and rapid endovascular procedures has significantly affected the mortality of subarachnoid haemorrhage (SAH). However, this is still high and amounts to $40 \%$. Moreover, about $30 \%$ of patients who survive the incident are severely disabled [1]. Therefore, to identify factors that are important for the prediction of aneurysm SAH outcomes is essential.

Nastasovic et al. conducted a prospective study and reviewed the data from more than 250 consecutive subjects with this life-threatening condition [2]. They used the Glasgow Outcome Score (GOS) for SAH prognosis assessment.

Aneurysm SAH is a complex disorder that involves damage to the central nervous system at different levels and multiple organ failure. Mortality in SAH is related to a substantial number of pathological conditions such as cerebral vasospasm (with secondary delayed cerebral ischaemia), recurrent subarachnoid haemorrhage (sometimes associated with intraventricular/ parenchymal bleeding), malignant intracranial hypertension, hydrocephalus, seizures, gastrointestinal bleeding, anaemia, neurogenic pulmonary oedema, cardiac complications, glucose abnormalities, electrolytes imbalance (mostly low or high sodium levels), and thromboembolic events [3].

Clearly therefore, designing a reliable study that would cover all potential factors affecting prognosis in this critical illness is challenging.
Numerous scientific reports have investigated SAH outcomes with regards to morphologic parameters of a ruptured aneurysm such as shape and flow angle, and especially its size [4]. Other studies have considered the clinical assessment of the initial neurological condition of patients estimated using the Hunt and Hess, Fisher, or World Federation of Neurosurgical Societies (WFNS) rating scales [5]. Some articles have analysed the role of various biochemical parameters in aneurysm SAH outcome estimation [6].

The article by Nastasovic et al. is very interesting because the researchers explored various clinical, selected biochemical, and aneurysm-associated factors all together. They observed patients for three months, finally describing the independent predictive factors of an unfavourable aneurysm SAH outcome to be high systolic blood pressure (SBP), high heart rate on hospital admission, and aneurysm re-rupture.

Intuitively, these parameters look familiar, especially given that premorbid hypertension is a well-recognised prognostic feature in this devastating disease. Only a few publications have pointed directly to the role of SBP measured at the beginning of hospitalisation in aneurysm SAH prognosis [7]. Recently, the SBP at admission and its variability were investigated in a clinical trial [8]. It is also very hard to find any article about heart rate as a factor critical to SAH outcome (estimated by GOS), and the revelation of this phenomenon by the team from Belgrade seems fundamental. 
Over and above the methodological constraints and study limitations mentioned by the authors, the article might have a valuable impact on routine clinical practice, not only in terms of decision-making but also by informing patients and their relatives about the nature of this critical illness. It certainly helps them to arrange assistance, anticipate the quality of life, and plan the near future.

In summary, the findings of Nastasovic et al. suggest major potential approaches (with the elaboration of relevant neurocritical care protocols) regarding reducing aneurysm SAH complications to be aneurysm re-bleeding prevention and cardiovascular system support. Hopefully in this way we can decrease aneurysm SAH mortality rates even more.

\section{Conflict of interest: None}

\section{References}

1. Rinkel GJ, Algra A. Long-term outcomes of patients with aneurysmal subarachnoid haemorrhage. Lancet Neurology. 2011; 10(4): 349-356.
2. Nastasovic T, Milakovic B, Stosic M, et al. Predictors of unfavorable outcome in aneurysmal subarachnoid hemorrhage. Neurol Neurochir Pol; 2019; 53(6): 421-427, doi: 10.5603/PJNNS.a2019.005.

3. Subarachnoid Hemorrhage. Muehlschlegel S. Continuum Minneap Minn. 2018; 24(6): 1623-1657.

4. Tai J, Liu J, Lv J, et al. Risk factors predicting a higher grade of subarachnoid haemorrhage in small ruptured intracranial aneurysm (< 5 mm). Neurol Neurochir Pol. 2019; 53(4): 296-303, doi: 10.5603/ pjnns.a2019.0029.

5. van Donkelaar CE, Bakker NA, Veeger NJ, et al. Prediction of outcome after subarachnoid hemorrhage: timing of clinical assessment. J Neurosurg. 2017; 126(1): 52-59, doi: 10.3171/2016.1.JNS152136, indexed in Pubmed: 27035175.

6. Hong C, Tosun C, Kurland D, et al. Biomarkers as outcome predictors in subarachnoid hemorrhage - a systematic review. Biomarkers. 2014; 19(2): 95-108, doi: 10.3109/1354750x.2014.881418.

7. Rosengart AJ, Schultheiss KE, Tolentino J, et al. Prognostic factors for outcome in patients with aneurysmal subarachnoid hemorrhage. Stroke. 2007; 38(8): 2315-2321, doi: 10.1161/STROKEAHA.107.484360, indexed in Pubmed: 17569871.

8. Yang M, Pan X, Liang Z, et al. Association between blood pressure variability and the short-term outcome in patients with acute spontaneous subarachnoid hemorrhage. Hypertens Res. 2019; 42(11): 1701-1707, doi: 10.1038/s41440-019-0274-y, indexed in Pubmed: 31171841. 